\title{
Improving diagnostic accuracy of skin biopsies
}

Paul Stevenson, Karl Rodins

\section{Background}

The skin biopsy is a simple but essential clinical skill of the general practitioner. Performed properly, it can be quick and comfortable for the patient, and yield a very high level of diagnostic information. Performed incorrectly, it can lead to delays in diagnosis and treatment for the patient.

\section{Objective}

This article reviews some simple but effective steps the clinician can take to ensure proper technique and maximise the diagnostic accuracy of their skin biopsies.

\section{Discussion}

Diagnostic accuracy can be improved through optimal selection of biopsy site, correct biopsy technique, requesting ancillary tests where appropriate, proper handling of specimens, and providing detailed clinical information for the dermatopathologist.
THE SKIN BIOPSY is an essential tool for the management of skin conditions. Performed properly, it can help establish a diagnosis and guide appropriate treatment. The treating clinician can dramatically improve the diagnostic accuracy of biopsy specimens by:

- choosing optimal biopsy site

- performing correct biopsy technique

- obtaining adequate tissue sample

- where indicated, taking additional biopsy samples for ancillary tests

- ensuring proper handling of biopsy specimen and using the correct transport medium

- providing detailed clinical information for the dermatopathologist on the request form.

The most common biopsy types are punch, shave, excisional and incisional. Each technique has its own advantages and yields different information.

\section{Choosing a lesion and site to biopsy}

The choice of biopsy site is particularly important in inflammatory conditions (rashes) where multiple lesions and/or skin sites may be available for biopsy. Generally, established lesions with the most primary inflammatory change should be chosen, as early lesions may reveal nonspecific features. ${ }^{1-3}$ An exception to this is blistering (bullous) disorders, pustular eruptions and suspected vasculitis, where early lesions biopsied within 48 hours of appearance show more specific features on histopathology. ${ }^{1,2,4,5}$

Older lesions with secondary skin changes such as crusting, scarring, regeneration and infection should be avoided, as they will have a lower yield..$^{1-3}$ Avoid biopsying lesions that have been excoriated, traumatised or recently treated, as the underlying pathological process will be obscured. ${ }^{3}$

For small, inflammatory lesions $(<4 \mathrm{~mm})$, a punch excision could be performed. For large inflammatory lesions, choose the edge of an expanding lesion, the area of most colour change, or the thickest portion of the lesion. ${ }^{1-3}$ Annular plaques should be biopsied at the elevated edge of the lesion. ${ }^{6}$

With an ulcer or ulcerated lesion, include a portion of normal adjacent tissue, because tissue from the ulcer bed alone often yields nonspecific findings. Best practice is to perform an incisional biopsy of the junction of ulcer and adjacent normal tissue. ${ }^{4,7}$

Multiple biopsies should be considered where the rash has a polymorphic appearance, selecting sites of varying morphology. ${ }^{4,6}$ Some dermatoses also require additional biopsies for ancillary tests (refer to discussion below).

Selecting a biopsy site is a balance between choosing representative tissue and avoiding problem-prone areas. Certain sites on the body should be avoided when other sites are available. Where possible, avoid cosmetically sensitive areas, such as the face and areas prone to hypertrophic scarring such as shoulders, chest and breasts. ${ }^{1-3}$ Avoid biopsying the legs and foot as venous stasis changes can affect interpretation and healing is often poor, especially in the elderly, diabetics and patients with vascular insufficiency. ${ }^{2}$ The axilla and groin are higher risk sites for infection and should be avoided. ${ }^{3}$ Biopsies of bony prominences or pressure-bearing areas can have interpretive challenges and are more likely to be traumatised during movement. ${ }^{6}$ Generally, the thighs, abdomen, back and arms are good areas to biopsy. ${ }^{2,6}$ Table 1 provides a summary of biopsy recommendations for common dermatological presentations. 
Table 1. Recommended biopsy technique for specific clinical presentations ${ }^{1,3-5,8,12}$

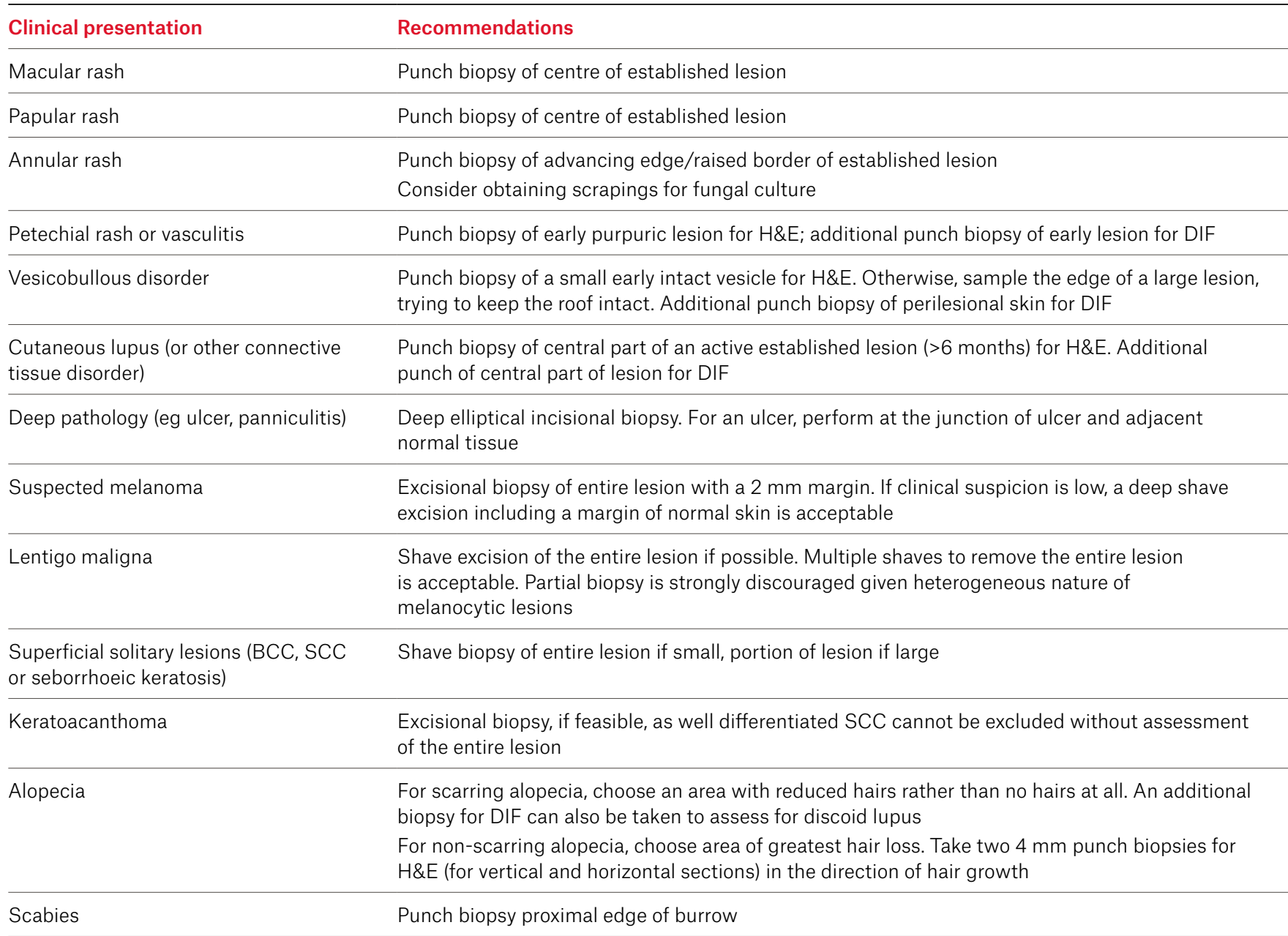

BBC, basal cell carcinomas; DIF, direct immunofluorescence; H\&E, haematoxylin and eosin; SCC, squamous cell carcinomas

\section{Preparation of site and anaesthesia}

The essential equipment required for biopsies is listed in Table 2. Begin by marking out the biopsy site with a surgical marker as it may become obscured after injection of local anaesthetic. For most punch or shave biopsies, $0.5 \mathrm{~mL}$ of local anaesthetic is sufficient. Too much anaesthetic can distort the histological appearances and simulate dermal oedema. ${ }^{7}$ For large excisional or incisional biopsies, infiltrate a few millimetres outside the marked excision site to anaesthetise where sutures will be thread. Lignocaine $1 \%$ is the most commonly used local anaesthetic agent. It also comes mixed with adrenaline in a 1:100,000 concentration, which prolongs anaesthesia effect and reduces bleeding. ${ }^{8}$

Clean the skin with an antiseptic agent such as isopropyl alcohol, chlorhexidine or povidone-iodine solution. Be gentle when cleaning the skin surface so as not to disturb the overlying scale, as keratin layers can sometimes contain diagnostic information. ${ }^{7}$ Chlorhexadine should not be used around ears due to risk of ototoxicity or around eyes due to corneal irritation or ocular injury. ${ }^{9}$

\section{Punch biopsy}

Punch biopsy is the preferred method of sampling for most inflammatory dermatoses because it allows the pathologist to examine all layers of skin from the epidermis through to the top layer of the subcuticular fat. For most punch biopsies, a $4 \mathrm{~mm}$ diameter punch provides adequate tissue sample for histopathology assessment. ${ }^{5,6,10}$ Smaller punch biopsies risk yielding insufficient tissue for accurate diagnosis and should be reserved for cosmetically sensitive areas. ${ }^{10}$ Consider a $5 \mathrm{~mm}$ punch in dermatoses with atypical features. ${ }^{6}$

For most inflammatory conditions, penetrating the punch instrument through reticular dermis into the subcutaneous fat is a sufficient depth. A feeling of 'giving way' marks the point of reaching the subcutaneous fat. Most punch instruments 
Table 2. Equipment for biopsies ${ }^{1,3-5,8,12}$

\begin{tabular}{|c|c|}
\hline Equipment & Comments \\
\hline \multicolumn{2}{|l|}{ Surgical marking pen } \\
\hline Skin preparation solution & Isopropyl alcohol, chlorhexidine, or povidone-iodine \\
\hline \multicolumn{2}{|l|}{ Gauze } \\
\hline \multicolumn{2}{|l|}{ Sterile drape } \\
\hline Gloves & Non-sterile is sufficient for shaves or punches \\
\hline Local anaesthesia & $\begin{array}{l}1 \% \text { lignocaine }+/-1: 100,000 \text { adrenaline. Sodium bicarbonate in a } 1: 9 \text { ratio can be } \\
\text { added to lignocaine to reduce pain }\end{array}$ \\
\hline \multicolumn{2}{|l|}{$3 \mathrm{ml}$ or $5 \mathrm{ml}$ syringe } \\
\hline \multicolumn{2}{|l|}{ Drawing up needle 18 or 21 gauge } \\
\hline Injecting needle 25 to 30 gauge & Pain can be minimised with slow injection of anaesthetic and a small-gauge needle \\
\hline \multicolumn{2}{|l|}{ Biopsy instrument: } \\
\hline - Punch (4 $\mathrm{mm}$ or $5 \mathrm{~mm})$ & For punch biopsy \\
\hline - Flexible shave instrument or scalpel blade & For shave biopsy \\
\hline - Scalpel holder + No. 15 scalpel blade & For excisional or incisional biopsy \\
\hline
\end{tabular}

Closure instruments (punch/excision/incision):

- 3.0-5.0 non-absorbable sutures

Absorbable sutures may also be required for deep excisions/incisions in areas of high

- Forceps skin tension

- Needle holder

- Scissors

Specimen containers in transport medium

$10 \%$ formalin for routine haematoxylin and eosin microscopy

Sterile urine jar with saline gauze or Michel's medium for direct immunoflourescence

Sterile jar for microbiological culture

Haemostatic agents

Aluminium chloride or aluminium sulphate with cotton bud applicators

Electrocauterisation device

White soft paraffin

Adhesive dressings

have a plastic hub that stops the punch going too deep.

Some sources recommend rotating the punch instrument in one direction as twisting back and forth may shear off fragile epidermis from dermis or increase the risk of 'double-cutting' the surface. ${ }^{1}$ However, if gentle care is taken then twisting is permissible, in our view.

Once sufficient depth is reached, remove the punch instrument and apply downward pressure either side of the wound to elevate the core for its edges. Lift the biopsy core with a hypodermic needle or very gently with a pair of forceps to prevent crush injury, which may affect interpretation. Free the specimen from the subcutaneous fat with iris scissors or scalpel blade.

In our view, punches $4 \mathrm{~mm}$ or more usually require closure with one or more interrupted sutures and will heal with a linear scar. Applying tension to the skin perpendicular to lines of least skin tension with your non-dominant hand during the procedure will leave an oval-shaped wound that is easier to suture closed. Punches $3 \mathrm{~mm}$ or less can usually heal with secondary intention without sutures.
Partial punch biopsies should not be performed on pigmented lesions, because the partial biopsy specimen may not represent areas of atypia or malignancy. Margin assessment is also more difficult to appreciate with punch biopsies because of how the pathology technician may dissect the specimen. ${ }^{8}$

\section{Shave biopsy}

Shave biopsy is the preferred method for solitary lesions that are raised or where pathology is confined to the epidermis. ${ }^{1-5,10}$ They are generally considered appropriate 
for superficial basal cell carcinomas (BCC) and squamous cell carcinomas (SCC). ${ }^{11,12}$ The ideal shave biopsy depth requires practice, and a balance between diagnostic accuracy and cosmetic outcome. Too superficial will be inadequate tissue for diagnosis. Too deep will delay healing and increase scarring. A sample to the level of the superficial dermis is usually sufficient. ${ }^{2,7,12}$

Shave biopsy is most easily performed using a flexible, gripped blade. Pathology labs often provide these consumables for free. Otherwise, a size 15 scalpel blade or scissors may be used. Make your first cut at 45 degrees angle to the skin at the edge of the lesion, moving the blade in a perpendicular motion. ${ }^{12}$ Once sufficient depth is achieved, flatten the blade to 0 degrees and continue moving the blade from side to side until the lesion is removed.

Haemostasis can be achieved using pressure, aluminium chloride $20 \%$ in alcohol ('Driclor'), aluminum sulphate in aqueous solution ('Stingose'), or electrocauterisation. ${ }^{12,13}$ Caution is advised when using electrocauterisation subsequent to aluminum chloride in alcohol due to its flammable nature.

Wounds heal by secondary intention and no sutures are required. Aluminum chloride solution can be applied with a cotton-tipped applicator several times with concomitant pressure. This generally scars less than electrocauterisation and is less cumbersome.

Curettage is a form of shave biopsy resulting in a fragmented specimen that hampers histopathological assessment. If using curettage to treat a lesion, we recommend sampling the lesion with a standard shave biopsy for diagnosis prior to curettage.

\section{Excisional and incisional biopsy}

An excisional biopsy is an appropriate technique for suspected melanomas, subcutaneous or deep dermal tumours, and deep inflammatory processes. ${ }^{2,4,7,8}$ It requires more time and skill than other biopsy techniques, but yields more tissue for the dermatopathologist and allows for multiple studies if required. ${ }^{1}$ The biopsy is performed like any standard excision by removing an elliptical piece of skin with a scalpel blade and closuring the wound with sutures. For a pigmented lesion, this should include the entire lesion with a $2 \mathrm{~mm}$ margin of normal skin. ${ }^{14}$ Ultimately, the excision must go to the level of subcutaneous fat for optimal histological diagnosis. ${ }^{2,4,7,8,15}$

Incisional biopsy is appropriate for deep inflammatory processes (eg pannicultis), ulcers, porokeratosis, cutaneous lymphoma and medium-vessel vasculitis..$^{2,4,7,8}$ It may also be appropriate where a large shave biopsy would leave an unacceptable scar on a cosmetically sensitive area, or where a punch biopsy does not provide sufficient representative tissue of the lesion. ${ }^{2}$ The technique is similar to an excisional biopsy, except only a portion of the lesion is biopsied and the sample should include a $1 \mathrm{~mm}$ area of normal adjacent skin at one end of the ellipse. ${ }^{7,8}$ The ellipse does not need to be as wide as an excision, but it should extend deep into the subcutaneous tissue to sample fatty tissue..$^{2,4,7,8,15}$

\section{The pathology request form and ancillary tests}

Detailed completion of the pathology request form is one of the most overlooked but important aspects of the biopsy procedure. Detailed clinical information and an accurate macroscopic description of the skin lesion(s) will greatly aid the dermatopathologist with interpretation and assist in arriving at a diagnosis that correlates with the clinical picture. Without this, the pathologist may report differential diagnoses that do not match the clinical impression, leading to confusion and delaying correct management. ${ }^{5}$ Studies have shown that taking the time to fill out the pathology request form with sufficient clinical detail results in higher rates of correct diagnosis. ${ }^{16}$ Important clinical information to include on the request form is listed in Box 1.

If ancillary testing is required to assist with diagnosis, this should be stipulated on the request form, otherwise the technician may not prepare the specimen appropriately. The most commonly used ancillary tests in dermatopathology are direct immunofluorescence (DIF) and microbiological culture. ${ }^{7,8}$ A separate, additional biopsy specimen should be taken for ancillary testing if it is indicated. Microbiological culture should be considered if an infective process is suspected. ${ }^{5}$ Indications for DIF include bullous disorders, lupus erythematosus (or other connective tissue disease) and cutaneous vasculitis. ${ }^{4,5}$ In cutaneous lupus, the biopsy for DIF should be taken from the representative lesions. ${ }^{4}$ For vesiculobullous disorders, biopsies for DIF should be from perilesional skin (normal skin within $1 \mathrm{~cm}$ of an active lesion) because the immune deposits can degrade in forming a vesicle, resulting in a false negative result. ${ }^{4,7}$

The provision of digital images (both clinical and dermoscopic) to the dermatopathologist may aid in diagnosis, particularly in the diagnosis of atypical-appearing lesions or challenging melanocytic lesions. This could be considered routine practice in the digital age.

\section{Handling and transport of specimens}

Improper handling and transport of specimens can damage tissue samples and impair the accuracy of histopathology interpretation and diagnosis. Handle specimens carefully to minimise crush injury. Ensure the tissue specimen is

\section{Box 1. Key information to include on the pathology request form for any biopsy}

- Patient age and gender

- Precise anatomical site of the biopsy

- Morphological description of the lesion(s), including any evolution in appearance

- Distribution of lesion(s)

- Duration of lesion(s)

- Clinical impression and differential diagnosis

- Prior skin biopsy result(s) or dermatological diagnoses

- Medications if drug eruption is a differential diagnosis

- Other relevant patient clinical history 
placed in the correct transport medium for the test you are requesting. Haematoxylin and eosin (H\&E) light microscopy is the routine test for most skin biopsies and should usually be transported in $10 \%$ buffered formalin solution.

Tissue for DIF can be submitted in a sterile pot with saline-soaked gauze. Delays in specimen collection and processing can result in reduced diagnostic accuracy. Keep this in mind if performing a biopsy on a Friday afternoon and specimens will not reach the pathology lab until Monday. Tissue for DIF can also be submitted in a transport medium such as Michel's medium when there is less urgency in processing the specimen. Tissue for microbiological culture can be submitted fresh in a sterile container. If unsure, consult your pathology provider.

The sample pot(s) should be labelled with patient identification details, the precise anatomical body site of the biopsy, time and date of biopsy, and all information checked against the request form for consistency.

\section{Postoperative care}

Good postoperative care will reduce the risk of complications and improve the long-term cosmetic appearance. Risk factors for wound complications include immune-compromised patients, smoking, and biopsies in the axilla, groin or below the waist. ${ }^{10}$ Evidence supporting the routine use of oral antibiotics for biopsies is lacking. ${ }^{17}$ If the patient is immunosuppressed or the biopsy site is high risk for infection, a single dose of cephalosporin antibiotics (within three hours of procedure) may be considered..$^{15,17}$ Biopsy sites should be dressed with a moist occlusive dressing and paraffin ointment that should remain undisturbed for 24 to 48 hours. ${ }^{10,15,17,18,19}$ Thereafter, until re-epithelialisation is complete, wounds should be gently cleaned daily with warm water and reapplication of paraffin ointment with nonstick dressing. ${ }^{10,15,18,19}$ Topical antibiotics are no more effective than white soft paraffin..$^{18,19}$ Sutures, if present, should be removed in 5-7 days for the face, 12-14 days for back and legs, and 7-10 days for other areas generally. ${ }^{10,12,15}$
Follow-up should be arranged to review wounds and discuss biopsy results.

\section{Conclusion}

The skin biopsy is a simple but essential clinical skill of the general practitioner. Performed properly, it can be quick and comfortable for the patient, and yield a very high level of diagnostic information. If unsure how best to perform the biopsy or if the lesion is in a high-risk area, specialist referral may be indicated. Where the histological findings are inconsistent with the clinical suspicion, request a second opinion from the same pathology provider. Where the clinical and histopathological findings combined do not formulate a clear diagnosis, specialist referral to a dermatologist is recommended.

\section{Authors}

Paul Stevenson MD, Medical Officer, Princess Alexandra Hospital, Brisbane, Qld; Associate Lecturer, School of Medicine, Griffith University, Gold Coast, Qld; Associate Lecturer, Faculty of Medicine, University of Queensland, Brisbane, Qld. paul.stevenson@griffithuni.edu.au

Karl Rodins FACD, FRCPA, Specialist Dermatologist, Queensland Institute of Dermatology, Brisbane, QId

Competing interests: None

Provenance and peer review: Not commissioned, externally peer reviewed.

\section{References}

1. Alguire PC, Mathes BM. Skin biopsy techniques for the internist. J Gen Intern Med 1998;13(1):46-54.

2. Sleiman R, Kurban M, Abbas O. Maximizing diagnostic outcomes of skin biopsy specimens. Int J Dermatol 2013;52(1):72-78. doi: 10.1111/j.13654632.2012.05731.x.

3. Zuber TJ. Skin biopsy techniques: When and how to perform a punch biopsy. Consultant 2012;52(6):1-5.

4. Elston DM, Stratman EJ, Miller SJ. Skin biopsy: Biopsy issues in specific diseases. J Am Acad Dermatol 2016;74(1):1-16. doi: 10.1016/j. jaad.2015.06.033.

5. Sina B, Kao GF, Deng AC, Gaspari AA. Skin biopsy for inflammatory and common neoplastic skin diseases: Optimum time, best location and preferred techniques. A critical review. J Cutan Pathol 2009;36(5):505-10. doi: 10.1111/j.16000560.2008.01175.x.

6. Nischal U, Nischal Kc, Khopkar U. Techniques of skin biopsy and practical considerations. J Cutan Aesthet Surg 2008;1(2):107-11. doi: 10.4103/09742077.44174

7. Whitehead K. Cutaneous biopsies. Taringa: Sullivan Nicolaides Pathology, 2014. Available at www.snp.com.au/media/188842/cutaneous biopsies.pdf [Accessed 1 August 2017].
8. Harvey NT, Chan J, Wood BA. Skin biopsy in the diagnosis of inflammatory skin disease. Aust Fam Physician 2017;46(5):283-88.

9. Lai P, Coulson C, Pothier DD, Rutka J. Chlorhexidine ototoxicity in ear surgery, part 1 : Review of the literature. J Otolaryngol Head Neck Surg 2011;40(6):437-40.

10. Yang S, Kampp J. Common dermatologic procedures. Med Clin North Am 2015;99(6):130521. doi: 10.1016/j.mcna.2015.07.004.

11. Cancer Council Australia and Australian Cancer Network. Clinical practice guide: Basal cell carcinoma, squamous cell carcinoma (and related lesions) - A guide to clinical management in Australia. Sydney: Cancer Council Australia and Australian Cancer Network, 2008.

12. Pickett $H$. Shave and punch biopsy for skin lesions. Am Fam Physician 2011;84(9):995-1002.

13. Howe N, Cherpelis B. Obtaining rapid and effective hemostasis: Part I. Update and review of topical hemostatic agents. J Am Acad Dermatol 2013;69(5):659. doi: 10.1016/j.jaad.2013.07.014.

14. Cancer Council Australia, Australian Cancer Network, Ministry of Health New Zealand. Clinical practice guidelines for the management of melanoma in Australia and New Zealand. Wellington: Cancer Council Australia, Australian Cancer Network, Ministry of Health New Zealand, 2008.

15. Robinson J, Hanke CW, Sengelmann R, Siegel D (editors). Surgery of the skin: Procedural dermatology. Chapter 14: Skin biopsy techniques. Philadelphia: Elsevier Mosby, 2005; p. 203-12.

16. Comfere NI, Peters MS, Jenkins S, Lackore K, Yost K, Tilburt J. Dermatopathologists' concerns and challenges with clinical information in skin biopsy requisition form: A mixed-methods study. J Cutan Pathol 2015;42(5):333-45. doi: 10.1111/ cup. 12485 .

17. Wright TI, Baddour LM, Berbari EF, et al. Antibiotic prophylaxis in dermatologic surgery: Advisory statement 2008. J Am Acad Dermatol 2008;59(3):464-73. doi: 10.1016/j. jaad.2008.04.031.

18. Del Rosso JQ. Wound care in the dermatology office: Where are we in 2011? J Am Acad Dermatol 2011;64(3 Suppl):S1-7. doi: 10.1016/j. jaad.2010.10.038.

19. Nijhawan RI, Smith LA, Mariwalla K. Mohs surgeons' use of topical emollients in postoperative wound care. Dermatol Surg 2013;39(8):1260-63. doi: 10.1111/dsu. 
